\title{
Texture Analysis Based on Micro Primitive Descriptor (MPD)
}

\author{
Rasigiri Venkata lakshmi \\ Research Scalaor, Dept.of CSE, University College of Eng. JNTUK, Kakinada, 533003, India \\ Email: rasigiri.venkatalakshmi@gmail.com \\ Prof. E. Srinivasa Reddy and prof. K. Chandra Sekharaiah \\ Pricipal, University College of Eng \& Technology, ANU, Guntur, 522510, India \\ Professor, JNTU SIT, Hyderabad, 500 085, India \\ Email: \{edara_67@yahoo.com, chandrasekharaiahk@gmail.com\}
}

\begin{abstract}
Texture classification is an important application in all the fields of image processing and computer vision. This paper proposes a simple and powerful feature set for texture classification, namely micro primitive descriptor (MPD). The MPD is derived from the $2 \times 2$ grid of a motif transformed image. The original image is divided into $2 \times 2$ pixel grids. Each $2 \times 2$ grid is replaced by a motif shape that minimizes the local ascent while traversing the $2 \times 2$ grid forming a motif transformed image. The proposed feature set extracts textural information of an image with a more detailed respect of texture characteristics. The results demonstrate that it is much more efficient and effective than representative feature descriptors, such as Random Threshold Vector Technique (RTV) features and Wavelet Transforms Based on Gaussian Markov Random Field (WTBGMF) approach for texture classification.
\end{abstract}

Index Terms-Micro primitive descriptor, motif transformed image, texture classification.

\section{INTRODUCTION}

The major role of Texture analysis and classification in many image areas, such as geo-sciences and remote sensing, medical imaging, stone texture classification, fault detection, image document processing and image retrieval. Texture is an exterior arrangement formed by uniform or non-uniform repeated patterns. It includes four fundamental problems: classifying images based on texture content; segmenting an image into regions of homogeneous texture; synthesizing textures for graphics applications; and establishing shape information from texture cue [1]. Texture discrimination or classification is the basis for many applications in computer vision. Texture classification has long been an important task in computer vision [ 2, 3, 4] by which different regions of an image are identified based on texture properties. It has been applied widely in different areas, such as medical image analysis [5], remote sensing [6], and biometrics [7] Texture classification methods used can be categorized as statistical, geometrical, model based [2, 8, 9] and signal processing methods. Texture analysis aims at representing texture in a model that is invariant to changes in the visual appearance of the texture. The visual appearance of a single texture can change dramatically under the influence of, e.g., lighting changes and $3 \mathrm{D}$ rotations. Texture is characterized not only by the grey value at a given pixel, but also by the grey value pattern in an adjacent to the pixel.

At the beginning, extracting statistical feature to classify texture images, such as the co-occurrence matrix method [2] and the filtering based methods [10], is the main stream. Rotation invariance is a critical issue in many applications. In order to address it, many algorithms were proposed. Kashyap and Khotanzad [11] were among the first researchers to study rotationinvariant texture classification by using a circular autoregressive model. Later, many other models were explored, including the multiresolution autoregressive model [12], hidden Markov model [13], and Gaussian Markov random field [14].

The major step in description and classification of texture is study of patterns. For studying spatial structural and the textural characteristics of an image data various approaches are existed. [15], Fourier analysis for texture classification and noise removal [16, 17], fractal dimension for texture classification [18], variograms [19, $20,21,22]$ and calculating local variance for classification [23]. The most useful concept for dealing with regular patterns within image data is Fourier analysis. It has been used to clean out impair in radar data and to remove the special effects of regular undeveloped patterns in image data [24]. The basic fundamental tool for studying the regular patterns is local variance. It was carried out newly [25, 26]. Hence, the study of patterns still plays a significant area of research in classification, recognition and characterization of textures [27]. In [28], Ojala et al. proposed to use the local binary pattern (LBP) histogram for rotation invariant texture classification. LBP is a simple but efficient operator to describe local image patterns. Using a group of filter banks, Varma and Zisserman [29] proposed a statistical learning based algorithm, namely maximal response 8 (MR8), with which a rotation invariant texton library is first built from 
a training set and then an unknown texture image is classified according to its texton distribution. Zhenhua Guo et.al proposed a complex texton, complex response 8 (CR8)[30]. In this an 8-dimensional feature vector is extracted. After that, similar to MR8, a complex texton library is built from a training set by $k$-means clustering algorithm and then an texton distribution is computed for a given texture image[31]. In [31] proposed sparse representation (SR) method. In this A texton training dataset is first constructed by extracting patches in the training images, and then an over-complete dictionary of patch textons is learned from it under the SR framework By sparsely representing the texture image over the learned texton dictionary, a histogram of SR coefficients can be computed and used as features for texture classification. In [32] Laurens van proposed a 2D rotation method. In this method, image based textons are inclined to $2 \mathrm{D}$ rotations of the texture. It compares image-based textons with rotation-invariant textons based on spin images and polar Fourier features. The performance of this method is evaluated on the CUReT texture dataset for classification of Textures. Each of these methods depends upon how the texture features are selected for characterizes texture image. Whenever a new texture feature is derived it is evaluated whether it precisely classifies the textures or not. In the present paper, Textons are considered as micro primitive descriptor for texture classification. The different textons may form various image features. The present study attempted to classify various HSV-based color stone textures classification based on MPD histogram, which is different from the earlier studies. Based on the MPD the present paper evaluated a classification feature which is rotationally invariant.

The rest of the paper is organised as follows. Section 2 describes MPD detection method. The section 3 describes experimental results when the proposed method is applied. Comparison of the proposed method with other existing methods is discussed in section 5. The conclusions are given in section 5 .

\section{MPD DETECTION METHOD}

Various algorithms are proposed by many researchers to extract color, texture and other features. Color is the most distinguishing important and dominant visual feature. That's why color histogram techniques remain popular in the literature. The main drawback of this is, it lacks spatial information. Texture patterns can provide significant and abundance of texture and shape information. One of the features proposed by motifs patterns [33] called MPD, represents the various patterns of image which is useful in texture analysis. The proposed method consists of three steps which are listed below. In the first step of the proposed MPD evaluation, the color image is converted in to grey level image by using any HSV color model. The following section describes the RGB to HSV conversion procedure

Step1: RGB to HSV color model conversion In color image processing, there are various color models in use today. The RGB model is mostly used in hardware oriented application such as color monitor. In the RGB model, images are represented by three components, one for each primary color - red, green and blue. However, RGB color space is not sensitive to human visual perception or statistical analysis. Moreover, a color is not simply formed by these three primary colors. When viewing a color object, human visual system characterizes it by its brightness and chromaticity. The latter is defined by hue and saturation. Brightness is a subjective measure of luminous intensity. It embodies the achromatic notion of intensity. Hue is a color attribute and represents a dominant color. Saturation is an expression of the relative purity or the degree to which a pure color is diluted by white light. HSV color space is a non-linear transform from RGB color space that can describe perceptual color relationship more accurately than RGB color space. In this paper, HSV color space is adopted.

HSV color space is formed by hue $(\mathrm{H})$, saturation (S) and value $(\mathrm{V})$. Hue denotes the property of color such as blue, green, red, and so on. Saturation denotes the perceived intensity of a specific color. Value denotes brightness perception of a specific color. Thus it can be seen that HSV color space is different from RGB color space in color variations. When a color pixel-value in RGB color space is adjusted, intensities of red channel, green channel, and blue channel of this color pixel are modified. That means color, intensity, and saturation of a pixel is involved in color variations. It is difficult to observe the color variation in complex color environment or content. However, HSV color space separates the color into hue, saturation, and value which means observation of color variation can be individually discriminated. According to above descriptions about HSV color space, it can obviously observe that HSV color space can describe color detail than RGB color space in color, intensity and brightness. In order to transform RGB color space to HSV color space, the transformation is described as follows:

The transformation equations for RGB to HSV color model conversion is given below

$$
\begin{aligned}
& V=\max (R, G, B) \\
& S=\frac{V-\min (R, G, B)}{V} \\
& \mathrm{H}=\frac{\mathrm{G}-\mathrm{B}}{6 \mathrm{~S}} \quad \text { if } \quad \mathrm{V}=\mathrm{R} \\
& \mathrm{H}=\frac{1}{3}+\frac{\mathrm{B}-\mathrm{R}}{6 \mathrm{~S}} \text { if } \mathrm{V}=\mathrm{G} \\
& \mathrm{H}=\frac{1}{3}+\frac{\mathrm{R}-\mathrm{G}}{6 \mathrm{~S}} \text { if } \quad \mathrm{V}=\mathrm{B}
\end{aligned}
$$

Where R, G, B are Red, Green, and Blue normalized in value $[0,1]$. In order to quantize the range of the $H$ plane is normalized with value $[0,255]$ for extracting features specifically

Step2: motifs texton pattern detection Texton-based texture classifiers classify textures based on their texton frequency histogram. The textons are defined as a set of blobs or emergent patterns sharing a common property all over the image $[34,35]$. Based on the texton theory [34, 
35], texture can be decomposed into elementary units, the texton classes of colors, elongated blobs of specific widths, orientation and aspect ratios, and the terminators of these elongated blobs.

The concept of "texton" was proposed in [34] more than 20 years ago, and it is a very useful tool in texture analysis. In general, textons are defined as a set of blobs or emergent patterns sharing a common property all over the image; however, defining textons remains a challenge. In [35], Julesz presented a more complete version of texton theory, with emphasis on critical distances (D) between texture elements on which the computation of texton gradients depends. Textures are formed only if the adjacent elements lie within the D-neighborhood. However, this D-neighborhood depends on element size. If the texture elements are greatly expanded in one orientation, pre-attentive discrimination is somewhat reduced. If the elongated elements are not jittered in orientation, this increases the texton-gradients at the texture boundaries. Thus, with a small element size, such as, $2 \times 2$ texture discrimination can be increased because the texton gradients exist only at texture boundaries [35]. In view of this and for the convenience of expression, the $2 \times 2$ block is used in this paper for textons detection.

There are many types of textons in images. In this paper, each texton is treated as a MPD. We only define six special types of motifs [33] textons for texture analysis. The six motifs are defined over a $2 \times 2$ grid, each depicting a distinct sequence of pixels starting from the top left corner as shown in Fig.1. In Fig.1 the six motifs are denoted as Z, N, U, C, Gamma and Alpha respectively. Each grid is scanned from top-left and those pixels formed a texton. Reverse direction of motifs are also considered. So, a total of 12 texton patterns are considered for texture classification. The first top-left six motifs of a $2 \times 2$ grid are shown in Fig. 1 .

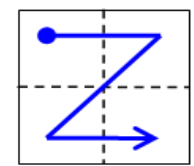

Z

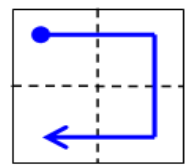

C

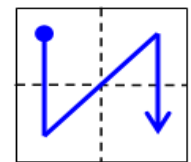

$\mathrm{N}$

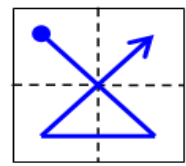

Gamma

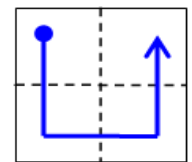

$\mathbf{U}$

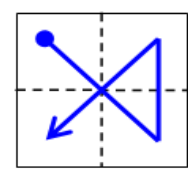

Alpha
Fig 1. Six motifs texton of a $2 \times 2$ grid

Once the motifs are selected, the original image is divided into $2 \times 2$ grids. Each of the $2 \times 2$ grids contains four pixel values i.e., $V_{1}, V_{2}, V_{3}$ and $V_{4}$. If the four pixel values of a $2 \times 2$ grid are distinct apply a suitable motif as in Fig. 1 otherwise $2 \times 2$ grid will be zero. The working mechanism of MPD detection for the proposed method is illustrated in Fig.2.

\begin{tabular}{|c|c|c|c|c|c|c|c|}
\hline 202 & 53 & 149 & 54 & 255 & 254 & 253 & 124 \\
\hline 78 & 55 & 84 & 52 & 57 & 190 & 186 & 250 \\
\hline 129 & 68 & 35 & 128 & 160 & 38 & 36 & 255 \\
\hline 183 & 29 & 140 & 68 & 54 & 31 & 144 & 182 \\
\hline 176 & 52 & 47 & 43 & 47 & 53 & 145 & 156 \\
\hline 145 & 38 & 61 & 45 & 47 & 62 & 140 & 176 \\
\hline 150 & 186 & 188 & 188 & 220 & 211 & 87 & 167 \\
\hline 99 & 196 & 189 & 174 & 155 & 159 & 151 & 106 \\
\hline
\end{tabular}

(a)

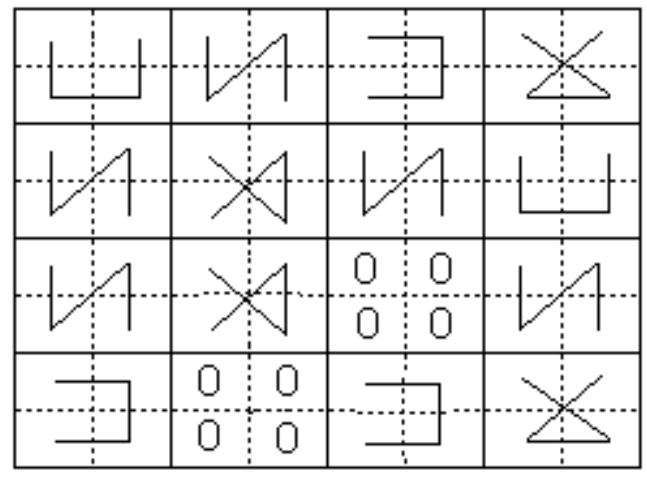

(b)

Fig 2. Example of motifs texton patterns a) $8 \times 8$ image b) motifs textons

Step 3: once the textons are identified The present paper evaluate the frequency occurrences of all six different textons (MPD) as shown in Fig.1 with different orientations. To have a precise and accurate stone texture classification, the present study considered sum of the frequencies of occurrences of all six different textons as shown in Fig. 1 on a $2 \times 2$ block.

\section{RESUlTS AND DiscUSSIONS}

The present paper carried out the experiments on two data sets. The dataset 1 consists of various Brick, Granite, Marble and Mosaic textures with resolution of $256 \times 256$ collected from Brodatz textures, VisTex and also from natural resources from digital camera. Some of texture images in dataset 1 are shown in the Fig. 3. The dataset 2 consists of various Brick, Granite, Marble and Mosaic textures with resolution of $200 \times 150$ collected from Outtex, CUReT database, and also from natural resources from digital camera. Some of texture images in dataset2 are shown in the Fig. 4.
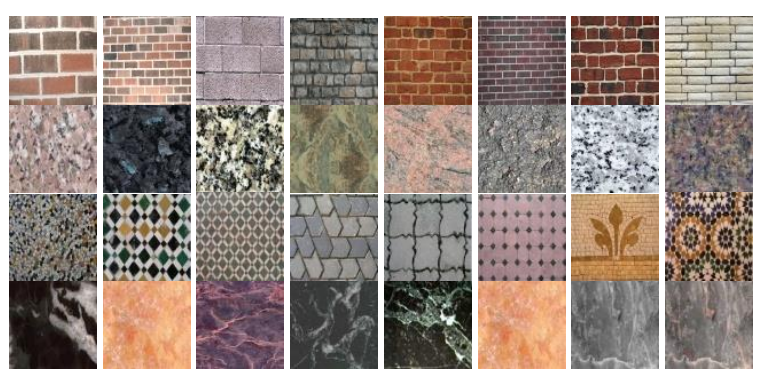

Fig 3. Input texture group of 8 samples of Brick, Granite, and Mosaic Marble with size of $256 \times 256$ 


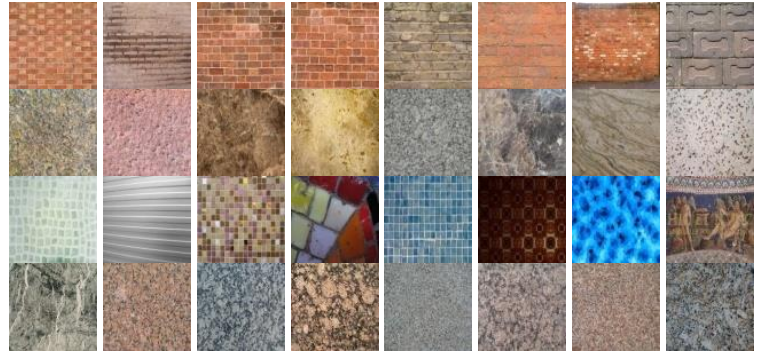

Fig 4. Input texture group of 8 samples of Brick, Granite, Mosaic, Marble with size of $200 \times 150$
The frequency of occurrence of MPD of Brick, Granite, Marble and Mosaic texture images in dataset1 are listed out in Table 1, 2, 3, and 4 respectively. The sum of frequency of occurrence of MPD of each input texture images in dataset1 are listed out in Table 5. The Table 1, 2, 3, 4, 5 and the classification graph of Fig.5, indicates a precise and accurate classification of the considered stone textures.

Tabble 1 Frequency occurrence of MPDs for brick textures in daraset1

\begin{tabular}{|c|c|c|c|c|c|c|c|}
\hline SNO & Texture name & $\mathbf{Z}$ & $\mathbf{N}$ & $\mathbf{U}$ & $\mathbf{C}$ & Alpha & Gama \\
\hline 1 & brick01 & 725 & 178 & 131 & 629 & 84 & 98 \\
\hline 2 & brick02 & 548 & 407 & 325 & 451 & 219 & 174 \\
\hline 3 & brick03 & 398 & 183 & 141 & 251 & 27 & 30 \\
\hline 4 & brick04 & 358 & 265 & 221 & 236 & 94 & 98 \\
\hline 5 & brick05 & 602 & 406 & 287 & 379 & 128 & 122 \\
\hline 6 & brick06 & 684 & 237 & 134 & 476 & 63 & 80 \\
\hline 7 & brick07 & 350 & 510 & 539 & 258 & 231 & 228 \\
\hline 8 & brick08 & 512 & 250 & 167 & 365 & 51 & 51 \\
\hline 9 & brick09 & 452 & 332 & 228 & 431 & 112 & 143 \\
\hline 10 & brick10 & 262 & 325 & 225 & 234 & 91 & 98 \\
\hline 11 & brick11 & 469 & 254 & 172 & 325 & 101 & 94 \\
\hline 12 & brick12 & 599 & 304 & 237 & 465 & 107 & 102 \\
\hline 13 & brick13 & 419 & 269 & 208 & 300 & 82 & 67 \\
\hline 14 & brick14 & 445 & 222 & 145 & 346 & 66 & 66 \\
\hline 15 & brick15 & 529 & 299 & 221 & 458 & 149 & 146 \\
\hline 16 & brick16 & 520 & 320 & 357 & 465 & 101 & 163 \\
\hline 17 & brick17 & 375 & 463 & 228 & 431 & 112 & 184 \\
\hline 18 & brick18 & 262 & 415 & 225 & 234 & 91 & 192 \\
\hline 19 & brick19 & 523 & 285 & 172 & 363 & 101 & 137 \\
\hline 20 & brick20 & 543 & 304 & 237 & 376 & 107 & 99 \\
\hline
\end{tabular}

Tabble 2 Frequency occurrence of MPD for granite textures in daraset1

\begin{tabular}{|c|c|c|c|c|c|c|c|}
\hline SNO & Texture name & Z & N & U & C & Alpha & Gama \\
\hline 1 & granite01 & 2235 & 2803 & 564 & 442 & 49 & 44 \\
\hline 2 & granite02 & 2124 & 2433 & 704 & 566 & 78 & 89 \\
\hline 3 & granite03 & 2244 & 3056 & 609 & 409 & 43 & 50 \\
\hline 4 & granite04 & 2633 & 2668 & 698 & 708 & 89 & 92 \\
\hline 5 & granite05 & 1958 & 3298 & 944 & 630 & 129 & 113 \\
\hline 6 & granite06 & 2752 & 2541 & 739 & 762 & 99 & 99 \\
\hline 7 & granite07 & 2402 & 2686 & 790 & 717 & 108 & 116 \\
\hline 8 & granite08 & 2450 & 2532 & 739 & 732 & 108 & 98 \\
\hline 9 & granite09 & 2468 & 2252 & 599 & 597 & 78 & 78 \\
\hline 10 & granite10 & 2379 & 2925 & 768 & 706 & 104 & 126 \\
\hline 11 & granite11 & 2378 & 2290 & 602 & 585 & 83 & 88 \\
\hline 12 & granite12 & 2194 & 2526 & 957 & 863 & 195 & 178 \\
\hline 13 & granite13 & 1639 & 3264 & 650 & 316 & 41 & 37 \\
\hline 14 & granite14 & 2382 & 2323 & 724 & 755 & 89 & 82 \\
\hline 15 & granite15 & 2121 & 3121 & 885 & 664 & 108 & 114 \\
\hline 16 & granite16 & 2164 & 2903 & 1062 & 876 & 181 & 173 \\
\hline 17 & granite17 & 2431 & 2460 & 711 & 723 & 83 & 83 \\
\hline 18 & granite18 & 2523 & 2572 & 857 & 815 & 149 & 114 \\
\hline 19 & granite19 & 2270 & 2651 & 719 & 656 & 64 & 67 \\
\hline 20 & granite20 & 2165 & 2535 & 1168 & 1023 & 270 & 264 \\
\hline
\end{tabular}


Tabble 3 Frequency occurrence of MPD for marble textures in daraset 1

\begin{tabular}{|c|c|c|c|c|c|c|c|}
\hline SNO & Texture name & Z & N & U & C & Alpha & Gama \\
\hline 1 & marble01 & 1361 & 2173 & 273 & 200 & 14 & 18 \\
\hline 2 & marble02 & 2124 & 1672 & 417 & 520 & 55 & 44 \\
\hline 3 & marble03 & 2236 & 1339 & 210 & 300 & 12 & 17 \\
\hline 4 & marble04 & 2002 & 2108 & 598 & 535 & 66 & 70 \\
\hline 5 & marble05 & 2021 & 1986 & 475 & 505 & 65 & 56 \\
\hline 6 & marble06 & 2044 & 1924 & 448 & 474 & 57 & 49 \\
\hline 7 & marble07 & 1578 & 1351 & 221 & 252 & 22 & 18 \\
\hline 8 & marble08 & 1376 & 2376 & 460 & 231 & 17 & 28 \\
\hline 9 & marble09 & 2021 & 2383 & 347 & 344 & 19 & 24 \\
\hline 10 & marble10 & 1543 & 1772 & 277 & 276 & 26 & 21 \\
\hline 11 & marble11 & 1428 & 1717 & 376 & 328 & 37 & 27 \\
\hline 12 & marble12 & 2793 & 1559 & 435 & 772 & 80 & 90 \\
\hline 13 & marble13 & 1073 & 1859 & 490 & 235 & 44 & 59 \\
\hline 14 & marble14 & 457 & 3247 & 406 & 80 & 6 & 8 \\
\hline 15 & marble15 & 1654 & 2465 & 817 & 546 & 89 & 112 \\
\hline 16 & marble16 & 1984 & 2210 & 567 & 511 & 71 & 63 \\
\hline 17 & marble17 & 2211 & 2096 & 614 & 708 & 100 & 116 \\
\hline 18 & marble18 & 2124 & 1672 & 417 & 520 & 55 & 44 \\
\hline 19 & marble19 & 2044 & 1924 & 448 & 474 & 57 & 49 \\
\hline 20 & marble20 & 2021 & 2383 & 347 & 344 & 19 & 24 \\
\hline
\end{tabular}

Tabble 4 Frequency occurrence of MPD for mosaic textures in daraset1

\begin{tabular}{|c|c|c|c|c|c|c|c|}
\hline SNO & Texture name & Z & N & U & C & Alpha & Gama \\
\hline 1 & mosiac01 & 774 & 790 & 370 & 376 & 131 & 139 \\
\hline 2 & mosiac02 & 799 & 817 & 348 & 343 & 104 & 106 \\
\hline 3 & mosiac03 & 891 & 688 & 318 & 378 & 109 & 119 \\
\hline 4 & mosiac04 & 664 & 577 & 350 & 326 & 129 & 137 \\
\hline 5 & mosiac05 & 860 & 857 & 535 & 493 & 237 & 218 \\
\hline 6 & mosiac06 & 953 & 891 & 385 & 414 & 252 & 252 \\
\hline 7 & mosiac07 & 1265 & 960 & 273 & 406 & 74 & 83 \\
\hline 8 & mosiac08 & 893 & 803 & 423 & 361 & 223 & 203 \\
\hline 9 & mosiac09 & 957 & 929 & 522 & 518 & 309 & 180 \\
\hline 10 & mosiac10 & 986 & 1005 & 533 & 487 & 206 & 186 \\
\hline 11 & mosiac11 & 905 & 897 & 537 & 554 & 257 & 290 \\
\hline 12 & mosiac12 & 1269 & 887 & 359 & 549 & 146 & 126 \\
\hline 13 & mosiac13 & 927 & 983 & 523 & 522 & 209 & 221 \\
\hline 14 & mosiac14 & 908 & 693 & 463 & 590 & 300 & 280 \\
\hline 15 & mosiac15 & 1078 & 910 & 435 & 549 & 148 & 161 \\
\hline 16 & mosiac16 & 940 & 911 & 403 & 392 & 120 & 107 \\
\hline 17 & mosiac17 & 793 & 729 & 378 & 374 & 154 & 150 \\
\hline 18 & mosiac18 & 911 & 752 & 431 & 561 & 217 & 218 \\
\hline 19 & mosiac19 & 1046 & 891 & 400 & 471 & 149 & 145 \\
\hline 20 & mosiac20 & 766 & 713 & 295 & 289 & 118 & 121 \\
\hline
\end{tabular}

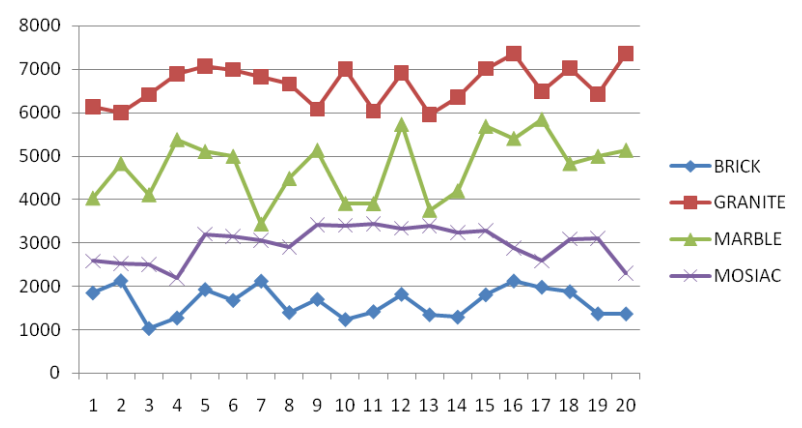

Fig 5. Classification graph of stone textures based on sum of the occurrences of texton
The Table 1, 2, 3, 4 and the classification graph of Fig.5, indicates that sum of frequency occurrences six texton features Z, N, U, C, Alpha and Gamma for Brick, Granite, Marble and mosaic in dataset1 textures are lying in-between 1030 to 2124,5947 to 7367,3442 to 5845 , and 2183 to 3440 respectively.

The frequency of occurrence of MPD of Brick, Granite, Marble and Mosaic texture images in dataset2 are listed out in Table 5, 6, 7, and 8 respectively. The sum of frequency of occurrence of MPD of each input texture images in dataset 1 are listed out in Table 10. 
Tabble 5 Frequency occurrence of MPD for Brick textures in daraset2

\begin{tabular}{|c|c|c|c|c|c|c|c|}
\hline SNO & Texture name & $\mathrm{Z}$ & $\mathrm{N}$ & $\mathrm{U}$ & $\mathrm{C}$ & Alpha & Gama \\
\hline 1 & brick01 & 856 & 644 & 269 & 497 & 50 & 45 \\
\hline 2 & brick02 & 763 & 737 & 354 & 382 & 66 & 78 \\
\hline 3 & brick03 & 837 & 663 & 248 & 498 & 88 & 77 \\
\hline 4 & brick04 & 934 & 566 & 304 & 380 & 46 & 69 \\
\hline 5 & brick05 & 857 & 643 & 382 & 388 & 76 & 94 \\
\hline 6 & brick06 & 638 & 862 & 317 & 401 & 57 & 51 \\
\hline 7 & brick07 & 876 & 624 & 253 & 556 & 55 & 52 \\
\hline 8 & brick08 & 971 & 529 & 275 & 464 & 61 & 91 \\
\hline 9 & brick09 & 965 & 535 & 304 & 516 & 49 & 78 \\
\hline 10 & brick10 & 864 & 636 & 344 & 456 & 98 & 85 \\
\hline 11 & brick11 & 833 & 667 & 288 & 362 & 41 & 26 \\
\hline 12 & brick12 & 569 & 931 & 237 & 524 & 51 & 62 \\
\hline 13 & brick13 & 597 & 903 & 209 & 458 & 38 & 40 \\
\hline 14 & brick14 & 913 & 587 & 206 & 517 & 55 & 59 \\
\hline 15 & brick15 & 949 & 551 & 229 & 528 & 56 & 51 \\
\hline 16 & brick16 & 569 & 931 & 276 & 466 & 31 & 48 \\
\hline 17 & brick17 & 930 & 570 & 205 & 637 & 50 & 61 \\
\hline 18 & brick18 & 759 & 741 & 207 & 584 & 65 & 69 \\
\hline 19 & brick19 & 860 & 640 & 355 & 385 & 81 & 77 \\
\hline 20 & brick20 & 843 & 657 & 242 & 560 & 86 & 64 \\
\hline 21 & brick21 & 781 & 719 & 234 & 419 & 24 & 29 \\
\hline
\end{tabular}

Tabble 6 Frequency occurrence of MPD for granite textures in daraset2

\begin{tabular}{|c|c|c|c|c|c|c|c|}
\hline SNO & Texture name & $\mathrm{Z}$ & $\mathrm{N}$ & $\mathrm{U}$ & $\mathrm{C}$ & Alpha & Gama \\
\hline 1 & granite01 & 598 & 902 & 458 & 571 & 173 & 181 \\
\hline 2 & granite02 & 530 & 970 & 465 & 450 & 158 & 191 \\
\hline 3 & granite03 & 687 & 813 & 455 & 425 & 141 & 123 \\
\hline 4 & granite04 & 692 & 808 & 441 & 458 & 137 & 123 \\
\hline 5 & granite05 & 751 & 749 & 517 & 432 & 136 & 145 \\
\hline 6 & granite06 & 988 & 512 & 391 & 601 & 117 & 122 \\
\hline 7 & granite07 & 909 & 591 & 523 & 458 & 149 & 158 \\
\hline 8 & granite08 & 805 & 695 & 508 & 483 & 167 & 180 \\
\hline 9 & granite09 & 802 & 698 & 425 & 578 & 123 & 146 \\
\hline 10 & granite10 & 569 & 931 & 414 & 492 & 149 & 143 \\
\hline 11 & granite11 & 731 & 769 & 220 & 590 & 96 & 102 \\
\hline 12 & granite12 & 845 & 655 & 412 & 496 & 133 & 144 \\
\hline 13 & granite13 & 972 & 528 & 488 & 533 & 177 & 147 \\
\hline 14 & granite14 & 864 & 636 & 397 & 464 & 112 & 104 \\
\hline 15 & granite15 & 762 & 738 & 503 & 483 & 152 & 165 \\
\hline 16 & granite16 & 876 & 624 & 454 & 428 & 174 & 170 \\
\hline 17 & granite17 & 850 & 650 & 408 & 367 & 178 & 92 \\
\hline 18 & granite18 & 694 & 806 & 270 & 549 & 96 & 90 \\
\hline 19 & granite19 & 846 & 654 & 433 & 438 & 107 & 96 \\
\hline 20 & granite20 & 921 & 579 & 481 & 327 & 123 & 80 \\
\hline 21 & granite21 & 945 & 555 & 418 & 456 & 116 & 96 \\
\hline 22 & granite22 & 936 & 564 & 529 & 469 & 151 & 164 \\
\hline
\end{tabular}


Tabble 7 Frequency occurrence of MPD for Marble textures in daraset2

\begin{tabular}{|c|c|c|c|c|c|c|c|}
\hline SNO & Texture name & $\mathrm{Z}$ & $\mathrm{N}$ & $\mathrm{U}$ & $\mathrm{C}$ & Alpha & Gama \\
\hline 1 & marble01 & 753 & 747 & 582 & 556 & 256 & 256 \\
\hline 2 & marble02 & 680 & 820 & 539 & 563 & 230 & 241 \\
\hline 3 & marble03 & 693 & 807 & 624 & 509 & 226 & 218 \\
\hline 4 & marble04 & 756 & 744 & 585 & 547 & 216 & 203 \\
\hline 5 & marble05 & 876 & 624 & 527 & 545 & 178 & 162 \\
\hline 6 & marble06 & 963 & 537 & 589 & 479 & 182 & 194 \\
\hline 7 & marble07 & 534 & 966 & 523 & 537 & 204 & 203 \\
\hline 8 & marble08 & 576 & 924 & 550 & 506 & 204 & 192 \\
\hline 9 & marble09 & 667 & 833 & 556 & 509 & 214 & 208 \\
\hline 10 & marble10 & 765 & 735 & 611 & 529 & 231 & 226 \\
\hline 11 & marble11 & 772 & 728 & 584 & 583 & 266 & 270 \\
\hline 12 & marble12 & 861 & 639 & 616 & 570 & 269 & 273 \\
\hline 13 & marble13 & 963 & 537 & 605 & 588 & 285 & 291 \\
\hline 14 & marble14 & 861 & 639 & 570 & 578 & 278 & 268 \\
\hline 15 & marble15 & 888 & 612 & 517 & 565 & 205 & 191 \\
\hline 16 & marble16 & 723 & 777 & 574 & 547 & 248 & 234 \\
\hline 17 & marble17 & 693 & 807 & 626 & 401 & 209 & 191 \\
\hline 18 & marble18 & 794 & 706 & 665 & 503 & 248 & 250 \\
\hline 19 & marble19 & 815 & 685 & 638 & 512 & 245 & 263 \\
\hline 20 & marble20 & 914 & 586 & 713 & 481 & 237 & 214 \\
\hline 21 & marble21 & 813 & 687 & 577 & 472 & 178 & 216 \\
\hline 22 & marble22 & 971 & 529 & 665 & 522 & 265 & 268 \\
\hline
\end{tabular}

Tabble 8 Frequency occurrence of MPD for Mosaic textures in daraset2

\begin{tabular}{|c|c|c|c|c|c|c|c|}
\hline SNO & Texture name & $\mathrm{Z}$ & $\mathrm{N}$ & $\mathrm{U}$ & $\mathrm{C}$ & Alpha & Gama \\
\hline 1 & mosiac01 & 896 & 604 & 239 & 290 & 56 & 58 \\
\hline 2 & mosiac02 & 675 & 825 & 257 & 255 & 23 & 33 \\
\hline 3 & mosiac03 & 824 & 676 & 213 & 218 & 8 & 22 \\
\hline 4 & mosiac04 & 867 & 633 & 82 & 72 & 3 & 1 \\
\hline 5 & mosiac05 & 610 & 890 & 276 & 199 & 12 & 15 \\
\hline 6 & mosiac06 & 725 & 775 & 135 & 169 & 16 & 19 \\
\hline 7 & mosiac07 & 795 & 705 & 226 & 282 & 49 & 62 \\
\hline 8 & mosiac08 & 595 & 905 & 135 & 202 & 6 & 9 \\
\hline 9 & mosiac09 & 535 & 965 & 92 & 91 & 6 & 6 \\
\hline 10 & mosiac10 & 634 & 866 & 236 & 238 & 37 & 37 \\
\hline 11 & mosiac11 & 916 & 584 & 318 & 288 & 46 & 24 \\
\hline 12 & mosiac12 & 827 & 673 & 119 & 140 & 8 & 6 \\
\hline 13 & mosiac13 & 809 & 691 & 188 & 223 & 43 & 42 \\
\hline 14 & mosiac14 & 907 & 593 & 260 & 305 & 27 & 33 \\
\hline 15 & mosiac15 & 506 & 994 & 155 & 157 & 6 & 7 \\
\hline 16 & mosiac16 & 632 & 868 & 2 & 392 & 5 & 3 \\
\hline 17 & mosiac17 & 681 & 819 & 331 & 175 & 77 & 72 \\
\hline 18 & mosiac18 & 781 & 719 & 41 & 38 & 5 & 5 \\
\hline 19 & mosiac19 & 786 & 714 & 178 & 125 & 9 & 8 \\
\hline 20 & mosiac20 & 985 & 515 & 188 & 251 & 94 & 70 \\
\hline 21 & mosiac21 & 927 & 573 & 165 & 203 & 10 & 14 \\
\hline 22 & mosiac22 & 765 & 735 & 80 & 90 & & 2 \\
\hline
\end{tabular}




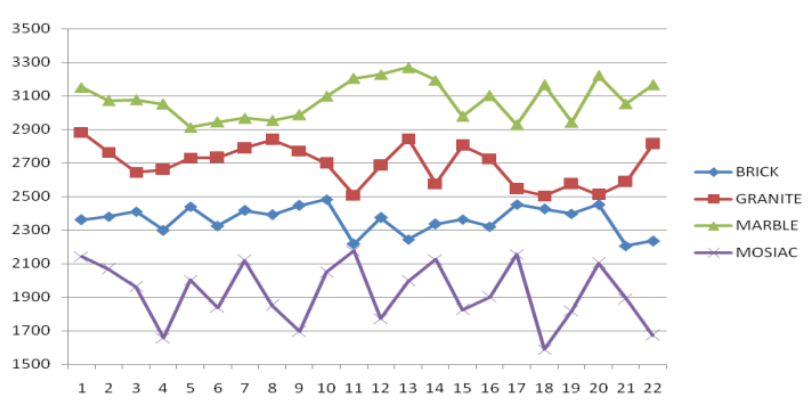

Fig.6: Classification graph of stone textures in dataset2 based on sum of the occurrences of texton

The Table 5, 6, 7, 8 and the classification graph of Fig.6, indicates that sum of frequency occurrences six texton features Z, N, U, C, Alpha and Gamma for Brick, Granite, Marble and mosaic in dataset 2 textures are laying in-between 2206 to 2484, 2505 to 2845,2912 to 3269 , and 1589 to 2176 respectively.

\section{COMPARISION WITH OTHER EXISTING METHODS}

The proposed motifs texton feature detection is compared with Random Threshold Vector (RTV) [36] and GMRF model on linear wavelets [37] methods. The above methods classified stone textures into three groups only. This indicates that the existing methods [36, 37] failed in classifying all stone textures. Further the present paper evaluated mean classification rate using $k-n n$ classifier. The percentage of classification rates of the proposed method and crashes methods $[36,37]$ are listed in table 11 . The table 11 clearly indicates that the proposed motifs texton feature detection outperforms the other existing methods and did not need any classification technique. Fig.7 shows the comparison chart of the proposed motifs texton feature detection with the other existing methods of Table 11.

Tabble 9 mean \% classification rate of the proposed and existing methods

\begin{tabular}{|c|c|c|c|}
\hline $\begin{array}{c}\text { Image } \\
\text { Dataset }\end{array}$ & $\begin{array}{c}\text { Random } \\
\text { Threshold Vector } \\
\text { Technique }\end{array}$ & $\begin{array}{c}\text { Wavelet } \\
\text { Transforms Based } \\
\text { on Gaussian } \\
\text { Markov Random } \\
\text { Field approach }\end{array}$ & $\begin{array}{c}\text { Proposed } \\
\text { Method } \\
\text { (MPD) }\end{array}$ \\
\hline Akar marble & 93.29 & 92.19 & 94.56 \\
\hline VisTex & 92.53 & 92.56 & 93.15 \\
\hline Outtex & 93.30 & 93.29 & 96.57 \\
\hline Brodatz & 93.59 & 92.86 & 95.06 \\
\hline CUReT & 92.76 & 91.76 & 95.97 \\
\hline
\end{tabular}

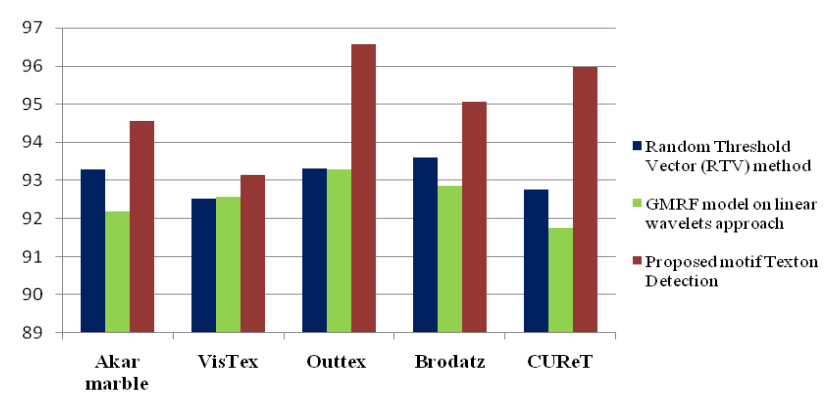

Fig 7. Comparison graph of proposed and existing systems

\section{CONCLUSIONS}

We proposed a new method, namely micro primitive descriptor (MPD), to describe image features for Texture classification which is rotationally invariant. The proposed MPD evaluates the relationship between the values of neighboring pixels. The proposed method has low time complexity and it is easy to implement. Textonbased texture classifiers form a new alternative to traditional texture classification approaches such as Markov Random Fields or filter bank models. The experiments were conducted on two datasets. The dataset consists of various Brick, Granite, Marble and Mosaic textures with resolution of $256 \times 256$ and $200 \times 150$ chosen from Brodatz, Vistex, Outtex, CUReT database, and also textured images from digital camera. From the graphs, it is shown that the frequency of MPD clearly classifies Brick, Marble, Granite and Mosaic textures. Recently Stone Texture Classification Based on Random Threshold Vector method classifies the Granite and Brick texture very clearly but this proposed method classifies 4 types of stone textures very clearly.

\section{ACKNOWLEDGMENT}

The authors wish to thank to all who are supported us while working in this research paper

\section{REFERENCES}

[1] Bovik, A. C., Clark, M. and Geisler, W. S. "Multichannel texture analysis using localized spatial filters", IEEE Trans. Patt. Anal. Mach. Intell., 12, 1, pp. 55-73, 1990.

[2] R.M. Haralick, K. Shanmugam, I. Dinstein, "Texture features for image classification", IEEE Transactions on System Man Cybernat, Vol. 8, No. 6, 1973, pp. 610-621.

[3] Haralick, R. M. "Statistical and structural approaches to texture", Proc. of 4th Int. Joint Conf. Pattern Recognition, pp. 45-60, 1979.

[4] Vijaya Kumar, V., Raju, U.S.N., Chandra Sekaran, K. and Krishna, V. V. " A New Method of Texture Classification using various Wavelet Transforms based on Primitive Patterns", ICGST International Journal on Graphics, Vision and Image Processing, GVIP, Vol.8, Issue 2, pp. 21-27, 2008.

[5] Jia, W., Huang, D.S., Zhang, D.: Palmprint Verification Based on Robust Line Orientation Code. Pattern Recognition 41(5), 1504-1513 (2008).

[6] Anys, H., He, D.C.: Evaluation of Textural and Multi polarization Radar Features for Crop Classification. IEEE Transactions on Geoscience and Remote Sensing 33(5), 1170-1181 (1995).

[7] Ji, Q., Engel, J., Craine, E.: Texture Analysis for Classification of Cervix Lesions. IEEE Transactions on Medical Imaging 19(11), 1144-1149 (2000).

[8] M. Tuceyran and A.K. Jain, "Texture analysis, in Handbook of Pattern Recognition and Computer Vision", (2nd edition), World Scientific Publishing Co., Chapter 2.1, 1998, pp. 207-248.

[9] F. Argenti, L. Alparone, and G. Benelli, "Fast algorithms for texture analysis using co-occurrence matrices", IEE Proceedings-F, Vol. 137, No. 6, December 1990, pp. 443448.

[10] Randen, T., Husy, J.H.: Filtering for Texture Classification: Pattern Analysis and Machine Intelligence 
21(4), 291-310 (1999).

[11] Kashyap, R.L., Khotanzed, A.: A Model-based Method for Rotation Invariant Texture Classification. IEEE Transactions on Pattern Analysis and Machine Intelligence 8(4), 472-481 (1986).

[12] Mao, J., Jain, A.K.: Texture Classification and Segmentation Using Multiresolution Simultaneous Autoregressive Models. Pattern Recognition 25(2), 173188 (1992).

[13] Wu, W.R., Wei, S.C.: Rotation and Grey-scale Transform-invariant Texture Classification Using Spiral Resampling, Subband Decomposition, and Hidden Markov Model. IEEE Transactions on Image Processing 5(10), 1423-1434 (1996).

[14] Deng, H., Clausi, D.A.: Gaussian MRF Rotation-invariant Features for Image Classification. IEEE Transactions on Pattern Analysis and Machine Intelligence 26(7), 951-955 (2004).

[15] Richards, J. A. and Xiuping, J. (1999). Remote Sensing Digital Analysis: An Introduction. Germany: SpringerVerlag, vol.3, pp.363-363.

[16] Moody, A. and Johnson, D. M. (2001). Land-surface phonologies from AVHRR using the discrete Fourier transform. Remote Sens. Environ., vol. 75, pp. 305-323.

[17] Zhang, M., Carder, K. and Muller, karger. (1999). Noise reduction and atmospheric correction for coastal applications of landsat thematic-mapper imagery. Remote Sens. Environ., vol. 70, pp. 167-180.

[18] Burrough, P. A "Multiscale sources of spatial variation in soil, the application of fractal concepts to nested levels of soil variation”, Journal of Soil Sci., vol. 34, pp. 577-597. 1983.

[19] Atkinson, P. M. and Lewis, P. (2000). Geostatistical classification for remote sensing: An introduction. Comput. Geo. sci., Vol. 26, pp. 361-371.

[20] Curran, P. J. (1988). The Semivariogram in Remote Sensing: An Introduction. Remote Sens. Environ., vol. 24, pp. 493-507.

[21] Treitz, P. (2001). Variogram analysis of high spatial resolution remote sensing data: An examination of boreal forest ecosystems. Int. J. Remote Sens., vol. 22, pp. 38953900.

[22] Woodcock, C. E., Strahler, A. H. and Jupp, D. L. (1988). The use of variograms in remote sensing II: Real digital images. Remote Sens. Environ., vol. 25, pp. 349-379.

[23] Moody, A. and Johnson, D. M. (2001). Land-surface phenologies from AVHRR using the discrete fourier transform. Remote Sens. Environ., vol. 75, pp. 305-323.

[24] McCloy, K.R. (2002). Analysis and removal of the effects of crop management practices in remotely sensed images of agricultural fields. Int. J. Remote Sens., vol. 23, pp. 403-416.

[25] Peder, Klith Bocher. and Keith, R. McCloy. (2006). The Fundamentals of Average Local Variance: Detecting Regular Patterns. IEEE Trans. on Image Processing, vol. 15, pp. 300-310.

[26] Suresh A. and Vijaya Kumar V. et al. (2007). Texture Classification by Simple Patterns on Edge Direction Movements. International Journal of Computer Science and Network Security, vol. 7, no. 11, pp. 221-225.

[27] Suresh A. and Vijaya Kumar V. et al. (2008). Classification of Textures by Avoiding Complex Patterns. Journal of Computer Science, Science Publications, USA, vol. 4(2), pp.133-138.

[28] Varma, M., Zisserman, A.: A statistical Approach to Texture Classification from Single Images. International Journal of Computer Vision 62(1-2), 61-81 (2005).
[29] Ojala, T., Pietikäinen, M., Mäenpää, T.T.: Multiresolution Grey-scale and Rotation Invariant Texture Classification with Local Binary Pattern. IEEE Transactions on Pattern Analysis and Machine Intelligence 24(7), 971-987 (2002).

[30] Zhenhua Guo, Qin Li, Lin Zhang, Jane You, Wenhuang Liu, and Jinghua Wang, "Texture Image Classification Using Complex Texton”, ICIC 2011, LNAI 6839, pp. 98104, 2012. Springer-Verlag Berlin Heidelberg 2012.

[31] Jin Xie, Lei Zhang, Jane You and David Zhang , "TEXTURE CLASSIFICATION VIA PATCH-BASED SPARSE TEXTON LEARNING".

[32] Laurens van der Maaten a Eric Postma a "Texton-based Texture Classification"

[33] N. Jhanwar, S. Chaudhuri, G. Seetharaman, B. Zavidovique "Content based image retrieval using motif cooccurrence matrix", Image and Vision Computing 22 (2004) 1211-1220.

[34] Julesz B., - Textons, The Elements of Texture Perception, and their Interac-tions," Nature, vol.290 (5802): pp.91-97, 1981.

[35] Julesz B., -Texton gradients: the texton theory revisited," Biological Cybernet-ics, vol.54 pp.245-251, 1986.

[36] B.V. Ramana Reddy, M.Radhika Mani, B.Sujatha, and Dr.V.Vijaya Kumar "Texture Classification Based on Random Threshold Vector Technique", International Journal of Multimedia and Ubiquitous Engineering Vol. 5, No. 1, January, 2010.

[37] B.V. Ramana Reddy, M. Radhika Mani, and K.V. Subbaiah, "Texture Classification Method using Wavelet Transforms Based on Gaussian Markov Random Field" International Journal of Signal and Image Processing Vol.1-2010/Iss.1 pp. 35-39.

\section{Authors Profiles}

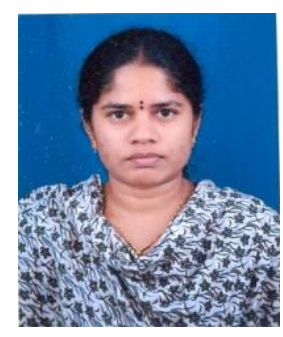

R. Venkata Lakshmi received B.Tech degree in Computer Science \& Engineering from Acharya Nagarjuna University. She received her M.Tech degree in Computer Science \& Engineering from Andhra University. Presently she is doing her Ph.D in JNTU kainada. Her Area of interest is Image Processing.

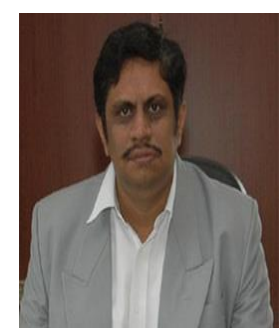

Prof. Sreenivas R.E. received the B.Tech degree in Electronics \& Communication Engienering from Nagarjuna University, India in 1988, M.S. degree from Birla Institute of Technology and Scince, India in 1997, M.Tech degree in Computer Science from Visveswaraiah Technological University, India in 2000 and Ph.D in computer science from Acharya Nagarjuna Univeristy, India in 2008. He is the senior member of IEEE and presented 11 papers in international conferences and 6 journal papers. His research interest includes image processing, biometrics and pattern recognition. He is currently supervising $2 \mathrm{Ph} . \mathrm{D}$ students who are working in different areas of image processing. 


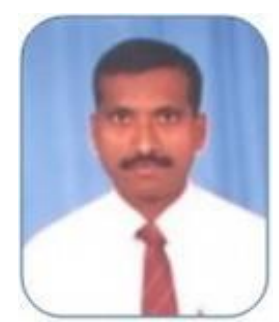

Dr. KChandrasekharaiah received he B.Tech degree from JNTU Hyderabad, M.Tech degree from JNTU Hyderabad and he got his Ph.D in Computer Science from IIT Madras. He received PostDoc in Software Engineering from University of Finland. He published more than 70 research papers in various National and Inter National Conferences and Journals. His research interest includes image processing, Software Engineering. He has 18 years of experience in teaching.

How to cite this paper: Rasigiri Venkata lakshmi, E. Srinivasa Reddy, K. Chandra Sekharaiah,"Texture Analysis Based on Micro Primitive Descriptor (MPD)", IJMECS, vol.7, no.2, pp.32-41, 2015.DOI: 10.5815/ijmecs.2015.02.05 